\title{
Attempts to Achieve Millennium Development Goals in a Rural Area of Rio de Janeiro: Deworming and Control of Schistosomiasis
}

\author{
Ricardo Pereira Igreja ${ }^{1 *}$, Magali Muniz Barreto², Marisa Silveira Soares ${ }^{2}$ \\ ${ }^{1}$ Departamento de Medicina Preventiva, Faculdade de Medicina, Universidade Federal do Rio de Janeiro, Rio de \\ Janeiro, RJ, Brazil \\ ${ }^{2}$ Lab. de Avaliação e Promoção da Saúde Ambiental, Instituto Oswaldo Cruz, Rio de Janeiro, Brazil
}

Corresponding Author: Ricardo Pereira Igreja, PhD, Associate Professor, Departamento de Medicina Preventiva, Faculdade de Medicina, Universidade Federal do Rio de Janeiro, Rio de Janeiro, RJ, Brazil. Tel: +55-21-25127812, Email: rpigreja@cives.ufrj.br

Received October 29, 2017; Accepted December 30, 2017; Online Published January 30, 2018

\begin{abstract}
Introduction: According to the World Health Organization (WHO), helminth control could contribute to achieving 7 Millennium Development Goals (MDGs). The present study evaluated the impact of deworming on the infection rate of schistosomiasis in Sumidouro, Brazil and the consequences for the MDGs.

Methods: The whole population of this area was invited to participate in the 2 stages of the study, the baseline from 2002 to 2003 and the follow-up from 2005 to 2006. Within this interval, no improvements to sanitation or access to safe water were undertaken. The statistical investigation was confined to the 164 people who were tested in both surveys. The diagnosis of parasitosis was based on coproscopy by Kato-Katz smears. Infected people were treated with a single oral dose of praziquantel.

Results: The infection rate of schistosomiasis dropped from $28.7 \%(n=47)$ to $6.7 \%(n=11)$. Among women of childbearing age, this rate decreased was from $27.8 \%(n=10)$ to $5.6 \%(n=2)$, and among children, it dropped from $14.6 \%(n=6)$ to $3.3 \%(n=1)$.

Conclusion: As there was no improvement in sanitation, deworming might have led to the reduced transmission of schistosomiasis. The drop in infection rate of schistosomiasis in children and women of childbearing age supports the argument that deworming would allow for the reduction of child mortality and the promotion of gender equality. A better future can also be foreseen, as no girl under 15 years of age was infected.

Keywords: Schistosomiasis, Praziquantel, Rio de Janeiro, Brazil
\end{abstract}

Citation: Igreja RP, Barreto MM, Soares MS. Attempts to achieve Millennium Development Goals in a rural area of Rio de Janeiro: deworming and control of schistosomiasis. Int J Travel Med Glob Health. 2018;6(1):30-33. doi:10.15171/ijtmgh.2018.06.

\section{Introduction}

In September, 2000, an unprecedented gathering of world leaders attended the United Nations (UN) Millennium Summit in New York, NY, USA and adopted the UN Millennium Declaration committing their nations to a new global partnership to halve the number of people living in poverty by 2015 . Several development targets and goals were set, including several in health that are collectively known as the Millennium Development Goals (MDGs). The UN Joint Monitoring Programme estimates that 2.6 billion people - $40 \%$ of the world's population - lack access to sanitation. Inadequate sanitation is one of the biggest contributing factors to child mortality under the age of 5 , and yet it remains the most neglected of the MDG sectors. Every year, 10 million children die before reaching their fifth birthday. Improved sanitation could bring the single greatest reduction in these deaths. ${ }^{1}$ Previous research in Brazil has shown that access to improved sanitation alone reduced the rate of childhood diarrhoea by up to $43 \% .^{2}$ Despite the strong evidence of the impact that increased access to sanitation would have on reducing child mortality, sanitation remains consistently overlooked in the formulation of development policy, and this has led to intense environmental contamination in many territories with helminths.

Schistosomiasis and soil-transmitted helminths (STH) are also deeply linked to the absence of sanitation. Human schistosomiasis is a major health problem in many parts of Africa, Asia, and America, and an estimated 200 million people in 76 countries are thought to be infected with one of the schistosome species that cause disease. ${ }^{3}$ There are

Copyright $\odot 2018$ The Author(s). This is an open-access article distributed under the terms of the Creative Commons Attribution License (http:// creativecommons.org/licenses/by/4.0), which permits unrestricted use, distribution, and reproduction in any medium, provided the original work is properly cited. 
still around 2.5 million to 6.3 million individuals harboring schistosomes in Brazil. ${ }^{4}$ In the state of Rio de Janeiro, some areas present low endemicity or isolated foci of Schistosoma mansoni.

Although the definitive resolution for schistosomiasis and STH is sanitation, deworming may be a useful tool in controlling this disease. Helminth control is a crucial, yet neglected, step towards improving public health and to reaching several of the MDGs. According to the World Health Organization (WHO), helminth control could contribute to achieving 7 of the $8 \mathrm{MDGs},{ }^{5}$ although just 3 of the 8 MDGs $(4,5$, and 6$)$ are directly related to health, concerning child health, maternal health, and disease control, respectively.

The present study evaluated the impact of deworming on the infection rate of schistosomiasis and the consequences for the MDGs in a rural area of Sumidouro (a county in the state of Rio de Janeiro, Brazil) that is strongly affected by helminths.

\section{Methods}

\section{Study Population/Recruitment}

This descriptive-cross sectional study was carried out in 2 contiguous rural localities (22 $\left.01^{\prime} \mathrm{S} 42^{\circ} 38^{\prime} \mathrm{W}\right)$ in Sumidouro, a county in the Brazilian state of Rio de Janeiro. In the present study, these villages were considered a unique area, since they have very similar physical and population characteristics. This area is surrounded by hills and has waterfalls and rapids as part of its main hydric collections. There are also some remains of the Brazilian Rain Forest on the mountaintops and in small parts along the stream margins. There was no sampling. The whole population of the study area was invited to participate in the study. At the invitation moment, informed consent forms were given to each of the adult subjects and to the parents or guardians of each child living in the locality. The name, age, gender, and address of each subject who decided to join the study were recorded. This is an interventional study carried out in 2 stages: the baseline from 2002 to 2003 and the follow-up from 2005 to 2006 . There were 255 participants at the baseline and 224 at the follow-up.

\section{Diagnosis and Treatment of Parasitosis}

The diagnosis of schistosomiasis was based on coproscopy by Kato-Katz method, ${ }^{6}$ using up to 3 faecal samples from each participant and 2 smears per faecal sample.

Each participant whose stool was found positive for $S$. mansoni eggs submitted to a general clinical examination. Adults and children were treated with a single oral dose of $40 \mathrm{mg} / \mathrm{kg}$ praziquantel. ${ }^{3}$ The tablets were administered with milk to minimize any gastrointestinal side effects. Within this interval, treatment was the only schistosomiasis control method undertaken in the villages, since there were no education measures, snail control, nor improvements to sanitation or access to safe water.

\section{Statistical Analysis}

Statistical investigation was confined to individuals who participated in both surveys. Estimates of schistosomiasis infection rate at the 2 surveys were compared, considering the whole population or individuals of different age or gender.
Comparisons used $\chi^{2}$ or Fisher exact tests, as appropriate. ${ }^{7}$ A $P$ value of less than 0.05 was considered statistically significant.

\section{Results}

From 164 individuals that participated in both surveys, 91 (55.5\%) were men and 73 (45\%) were women.

In the first survey, there were 41 (25\%) persons under the age of 15 years (counted as children), 80 (48.8\%) between 15 and 45 years of age, and $43(26.2 \%)$ over the age of 45 years. Three years later, 30 (18.3\%) people were under the age of 15 years, 83 (50.6\%) were between 15 and 45 years of age, and 51 $(31.1 \%)$ were over the age of 45 years. There were 36 women of childbearing age (between 15 and 45 years) each time.

The infection rate of schistosomiasis dropped from $28.7 \%$ $(\mathrm{n}=47)$ to $6.7 \%(\mathrm{n}=11)$. Of these 11 individuals, 6 had been treated in the first study and 5 were "new cases".

The infection rate of each specific group is shown in Table 1. As can be seen, in the more vulnerable groups, such as children and women, the infection rate dropped from $14.6 \%$ to $3.3 \%$ and from $20.5 \%$ to $2.7 \%$, respectively. Among women of childbearing age, the infection rate decreased from $27.8 \%$ to $5.6 \%$. No girl under 15 years of age was infected in 20052006.

All patients had intestinal or hepatointestinal forms of schistosomiasis, a milder form of the disease.

\section{Discussion}

The WHO estimates that helminth control could contribute to achieving 7 of the 8 MDGs: Goal 1 - Eradicate extreme poverty and hunger; Goal 2 - Achieve universal primary education; Goal 3 - Promote gender equality and empower women; Goal 4 - Reduce child mortality; Goal 5 - Improve maternal health; Goal 6 - Combat HIV/AIDS, malaria, and other diseases; Goal 8 - Develop a global partnership for development. ${ }^{5}$

Schistosome infection during childhood causes substantial growth retardation and anemia. Infected children may also have cognitive impairments and memory deficits. ${ }^{8}$ In the current study, the infection rate of schistosomiasis in children dropped from $14.6 \%$ to $3.3 \%$. This supports the argument that deworming would lead to the achievement of Goal 4 of the MDGs. Worm infection weakens very young children in ways that increase their vulnerability to infectious diseases. The benefits of regular deworming in this age group include improvements in iron stores, growth and physical fitness,

Table 1. Distribution of Infection Rate of Schistosomiasis

\begin{tabular}{lccc}
\hline \multirow{2}{*}{ Group } & \multicolumn{3}{c}{ Number (\%) of Subjects Infected in Each } \\
Group by Survey \\
\cline { 2 - 4 } & First & Second & $\boldsymbol{P}$ \\
\hline Men & $32(35.2)$ & $9(9.9)$ & $<0.05$ \\
Women & $15(20.5)$ & $2(2.7)$ & $<0.05$ \\
Women of childbearing age & $10(27.8)$ & $2(5.6)$ & $<0.05$ \\
Children & $6(14.6)$ & $1(3.3)$ & 0.11 \\
Girls & $3(15)$ & $0(0)$ & 0.17 \\
Boys & $3(14.3)$ & $1(6.7)$ & 0.44 \\
\hline
\end{tabular}


cognitive performance, and school attendance. ${ }^{9}$ Deworming in childhood leads to improvements in intellectual development that are related to income in adulthood.

Schistosome infection appears to have adverse effects on both maternal health and the fetus. ${ }^{8}$ A significant drop in helminth infection was seen in women of childbearing age, from $27.8 \%$ to $5.6 \%$. Furthermore, a better future can be foreseen, as no girl under the age of 15 years was infected. This could be a contribution to the achievement of Goal 3. For various reasons, anemia may be present in schistosomiasis. ${ }^{3}$ Abundant evidence shows that regular deworming reduces anemia in adolescent girls and women of childbearing age, thus preparing them for a healthier pregnancy. ${ }^{10}$

Some argue that treatment of sanitation-related disease is a significant drain on scarce financial resources among this section of society, and that the money spent on medicine and healthcare will be at the expense of food, education, and other essentials $^{1}$; however, worm control is possible with cheap and effective drugs. Praziquantel for schistosomiasis can be given as a single dose according to height, and it costs around $\$ 0.20$ USD for a school-aged child. An investment of as little as $\$ 3.5$ per child for helminth control translates to an extra year of schooling gained. ${ }^{10-13}$ Those are the reasons why the authors claim that in situations like that one studied herein, in which a population's needs have been neglected for too long and the environment is strongly contaminated with helminths, the benefits obviously justify the costs of efforts to adopt medication in addition to the improvements achieved in social services.

Indeed, as the current study was not undertaken in an area of high intensity of schistosomiasis, it cannot be predicted whether deworming would have the same effect on a more severely affected area. However, the current study has shown very positive results in controlling infection in the studied area of moderate intensity. It is possible that the interruption of schistosome transmission in high-risk areas will require more complicated, integrated control strategies, i.e. a combination of drug treatment, water management, snail control, and the control or treatment of sewage. ${ }^{14}$

\section{Conclusion}

Achieving the MDGs will require targeting areas and population groups that have clearly been left behind, like rural communities. In the current study, as there was no improvement to sanitation, deworming might have led to a reduced transmission of schistosomiasis. Although the authors emphasize the fact that treatment cannot replace improvements in sanitation, education, and other services, that drug delivery may be only a stopgap measure, and that the elimination of schistosomiasis will be a long-term process requiring a long-term investment, ${ }^{14}$ this study affirms that deworming is an essential tool for achieving the MDGs.

\section{Authors' Contributors}

RPI wrote the report, examined, and treated the patients. MMB participated in the study conception, design, and statistical analysis of data. MSS participated in the study conception, design, and the critical revision of the manuscript.
Research Highlights

\section{What Is Already Known?}

Schistosomiasis and soil-transmitted helminths (STH) are deeply linked to the absence of sanitation. Although the definitive resolution for schistosomiasis and STH is sanitation, deworming may be a useful tool in controlling this disease. According to the World Health Organization, helminth control could contribute to achieving 7 of the 8 Millennium Development Goals (MDGs), although just 3 of the $8 \mathrm{MDG}$ are directly related to health.

\section{What This Study Adds?}

Deworming with praziquantel resulted in the reduction in infection rates of schistosomiasis in children and in women of childbearing age in Sumidouro, as there was no improvement to sanitation during the study. A better future can be foreseen, as no girl under 15 years of age was infected after deworming. As the current study was not undertaken in an area of high intensity of schistosomiasis, it cannot be predicted whether deworming would have the same effect on a more severely affected area.

\section{Conflict of Interest Disclosures}

The authors declare that they have no conflicts of interest.

\section{Ethical Approval}

The study protocol was approved by the Ethics Committee on Research of the Oswaldo Cruz Foundation, in the city of Rio de Janeiro, license number 182/02.

\section{Funding/Support}

The current study was financed by Fiocruz, Brazil. The sponsor of the study had no role in the design, data collection, data analysis, data interpretation, or writing of the report.

\section{References}

1. Cumming $O$. The sanitation imperative: A strategic response to a development crisis. Desalination. 2009;248(1):8-13. doi:10.1016/j.desal.2008.05.031.

2. Barreto ML, Genser B, Strina A, et al. Effect of city-wide sanitation programme on reduction in rate of childhood diarrhoea in northeast Brazil: assessment by two cohort studies. Lancet. 2007;370(9599):1622-1628. doi:10.1016/s0140-6736(07)616389.

3. World Health Organization. Prevention and control of schistosomiasis and soil-transmitted helminthiasis: report of a WHO expert committee. WHO Technical Report Series 912. Geneva, Switzerland: WHO; 2002.

4. Igreja RP, Gusmao MF, Barreto MG, et al. A 15-year follow-up study on schistosomiasis in a low-endemic area in Rio de Janeiro State, Brazil. J Helminthol. 2010;84(3):229-233. doi:10.1017/ s0022149x09990575.

5. World Health Organization. The Millenium Development Goals. The evidence is in: deworming helps meet the Millenium Development Goals. http://whqlibdoc.who.int/hq/2005/WHO_ CDS_CPE_PVC_2005.12.pdf. Published 2005.

6. Katz N, Chaves A, Pellegrino J. A simple device for quantitative stool thick-smear technique in Schistosomiasis mansoni. Rev Inst Med Trop Sao Paulo. 1972;14(6):397-400.

7. Siegel S. Estatística Não-paramétrica para as Ciências do Comportamento. 1st ed. São Paulo: McGraw-Hill; 1975. 
8. Ross AG, Bartley PB, Sleigh AC, et al. Schistosomiasis. N Engl J Med. 2002;346(16):1212-1220. doi:10.1056/NEJMra012396.

9. Bethony J, Brooker S, Albonico M, et al. Soil-transmitted helminth infections: ascariasis, trichuriasis, and hookworm. Lancet. 2006;367(9521):1521-1532. doi:10.1016/s0140-6736(06)686534.

10. Thinking beyond deworming. Lancet. 2004;364(9450):19931994. doi:10.1016/s0140-6736(04)17521-1.

11. Davis A. Schistosomiasis. In: Cook GC, ed. Manson's Tropical Diseases. 20th ed. London: W B Saunders; 1996:1413-1456.

12. Raso G, N'Goran EK, Toty A, et al. Efficacy and side effects of praziquantel against Schistosoma mansoni in a community of western Cote d'Ivoire. Trans R Soc Trop Med Hyg. 2004;98(1):1827. doi:10.1016/S0035-9203(03)00003-8.

13. Igreja RP, Matos JA, Goncalves MM, Barreto MM, Peralta JM. Schistosoma mansoni-related morbidity in a low-prevalence area of Brazil: a comparison between egg excretors and seropositive non-excretors. Ann Trop Med Parasitol. 2007;101(7):575-584. doi:10.1179/136485907×229086.

14. King $\mathrm{CH}$. Toward the elimination of schistosomiasis. N Engl J Med. 2009;360(2):106-109. doi:10.1056/NEJMp0808041. 